\title{
STRONGLY PRIME NEAR-RINGS
}

\author{
by N. J. GROENEWALD
}

(Received 24th October 1985, revised 22nd July 1987)

\section{Introduction}

Strongly prime rings were introduced by Handelman and Lawrence [5] and in [2] Groenewald and Heyman investigated the upper radical determined by the class of all strongly prime rings. In this paper we extend the concept of strongly prime to nearrings. We show that the class $M$ of distributively generated near-rings is a special class in the sense of Kaarli [6]. We also show that if $N$ is any distributively generated nearring, then $U M(N), U M$ denotes the upper radical determined by the class $M$, coincides with the intersection of all the strongly prime ideals of $N$.

\section{Preliminaries}

Unless otherwise stated, all near-rings are zero-symmetric right near-rings. For undefined terminologies, we refer to [9].

Definition 1. Let $N$ be a near-ring. $N$ is called (right) strongly prime if and only if for every $0 \neq a \in N$ there exists a finite subset $F$ of $N$ such that $r(a F)=\{n \in N: a F n=0\}=0$. F is called an insulator of $a$ in $N$.

We now give the following alternative definition (c.f. [8] for corresponding definition for rings).

Definition 2. Let $N$ be a near-ring. $N$ is called (right) strongly prime if and only if every nonzero ideal $I$ of $N$ contains a finite subset $F$ such that $r(F)=0$.

The two definitions of strongly prime agree for the class of zero-symmetric near-rings.

The proof of this is based on the following observation and lemma.

Observation. Let $N$ be a zero-symmetric near-ring and $X \subset N$. The ideal generated by $X$ is the intersection of all ideals containing $X$ and can be obtained as follows:

Let $X_{0}=X \cup X N$

$X_{0}^{+}$be the normal subgroup of $N$ generated by $X_{0}$.

$$
\begin{aligned}
{\left[X_{0}^{+}\right]=\{z \in N \mid z} & \left.=c(a+b)-c a, a, c \in N, b \in X_{0}^{+}\right\} \\
X_{1} & =\left[X_{0}^{+}\right] \cup X_{0}^{+} .
\end{aligned}
$$


Repeat this process to obtain successively $X_{2}, X_{3}, \ldots$ Clearly $X_{0} \subset X_{1} \subset X_{2} \subset \ldots$ and it is straightforward to show that the ideal of $N$ generated by $X$ is precisely $\bigcup_{0}^{\infty} X_{i}$.

It is worth noting that an element of $X_{i}$ can be expressed by a formula involving only a finite number of terms involving expressions of the type $n+z x y-n$ where $n, z, y \in N, x \in X$.

Lemma 2.1. If $0 \neq a \in N$ and $a N=0$ then $\langle a\rangle N=0$.

Proof. $a N=0$ implies $a x N=0, z a N=0$ for all $x, z \in N$. Hence $X_{0} N=0$. Suppose $X_{i} N=0$. Then $\mathrm{n} \in N$ implies

$$
\begin{gathered}
\left(\sum(r+u-r)\right) n=\sum(r n+u n-r n)=0 \text { when } \pm u \in X_{i} \\
(r(a+u)-r a) n=r(a n+u n)-r a n=0 \text { when } \pm u \in X_{i} .
\end{gathered}
$$

Hence $X_{i+1} N=0$. It follows that $\langle a\rangle N=0$.

Corollary. If every ideal of $N$ contains a subset $F$ with $r(F)=0$ then for each $a \in N$, $a \neq 0$, there is $a \quad y \in N$ with $a y \neq 0$.

Theorem 2.2. The following are equivalent:

(i) $a \in \mathrm{N}, a \neq 0 \Rightarrow$ there is a finite subset $F$ of $N$ with $r(a F)=0$.

(ii) Every non-zero ideal of $N$ contains a finite subset $F$ with $r(F)=0$.

Proof. (i) $\Rightarrow$ (ii) is obvious.

Suppose (ii) and let $a \in N, a \neq 0$. Then $\langle a\rangle$, the ideal of $N$ generated by $a$, is non-zero and hence by the corollary there exists $y \in N$ with $a y \neq 0$ and a finite subset $G \subset\langle a y\rangle$ with $r(G)=0$. Let $X_{0}=\{a y\} \cup a y N$. $G$ is finite to $G \subseteq X_{j}$ for some $j$. Hence each element is obtained by applying a finite number of operations of the type

$$
\sum(r+u-r), u \in X_{j} \text { or } r(a+u)-r a, u \in X_{j} .
$$

Choose one such construction for each element of $G$. This set of constructions will involve a finite number of elements of $X_{0}$ of the form ays $s_{k}, s_{k} \in N$.

Let $G^{\prime}=\left\{a y, a y s_{k} \mid\right.$ these occur in the chosen construction of an element of $\left.G\right\}$.

Clearly $G^{\prime}$ is finite and $r\left(G^{\prime}\right) \subseteq r(G)=0$.

Then $H=\left\{z \mid a z \in G^{\prime}\right\}$ is an insulator of $a$ in $N$ and so (ii) $\Rightarrow$ (i).

We define an ideal $I$ of the near-ring $N$ to be strongly prime if and only if for every $x \notin I$, there is a finite subset $F$ of $\langle x\rangle$ such that for all $a \in N, F a \subseteq I$ implies $a \in I$.

It is now clear from the definition of a strongly prime near-ring and a strongly prime ideal that $N / I$ is a strongly prime near-ring if and only if $I$ is a strongly prime ideal. Furthermore, $N$ is strongly prime if $(0)$ is a strongly prime ideal. 
Lemma 2.3. If $N$ is a strongly prime near-ring, then $N$ is a prime near-ring.

Proof. Let $0 \neq A ; B \triangle N$. We show $A B \neq 0$. Since $A \neq 0$, there exists a finite subset $F$ of $A$ such that $r(F)=0$. Hence for each $0 \neq b \in B$ we have $F b \neq 0$. Therefore $A B \neq 0$.

We give the following example to show that in general a prime near-ring need not be a strongly prime near-ring.

Example 1. Consider the dihedral group $N$ with addition and multiplication defined as in Pilz ([9, p. 345 , number 11$])$ :

\begin{tabular}{lllllllll}
$\cdot$ & 0 & 1 & 2 & 3 & 4 & 5 & 6 & 7 \\
\hline 0 & 0 & 0 & 0 & 0 & 0 & 0 & 0 & 0 \\
1 & 0 & 1 & 0 & 1 & 0 & 1 & 1 & 0 \\
2 & 0 & 2 & 0 & 2 & 0 & 2 & 2 & 0 \\
3 & 0 & 3 & 0 & 3 & 0 & 3 & 3 & 0 \\
4 & 4 & 4 & 4 & 4 & 4 & 4 & 4 & 4 \\
5 & 4 & 5 & 4 & 5 & 4 & 5 & 5 & 4 \\
6 & 4 & 6 & 4 & 6 & 4 & 6 & 6 & 4 \\
7 & 4 & 7 & 4 & 7 & 4 & 7 & 7 & 4
\end{tabular}

$I=\{0,1,2,3\} \triangleleft N$. Furthermore $\{0,2\} \triangleleft I$ but $\{0,2\} \triangleleft N$. Clearly $N$ is prime. For each $F \subseteq I, F$ finite, we have $F \cdot 4=0$. Hence $r(F) \neq 0$ and $N$ not strongly prime.

In [7] Oswald defined a strictly prime near-ring as a near-ring $N$ such that if $A$ and $B$ are two $N$-subgroups of $N$ such that $A B=0$, then $A=0$ or $B=0$. When $N$ has a multiplicative identity or if $N$ is a d.g. near-ring, then $N$ is strictly prime if and only if for $a, b \in N, a N b=0$ implies $a=0$ or $b=0$.

Proposition 2.4. If $N$ is distributively generated or has an identity then $N$ is strongly prime if $N$ is strictly prime with D.C.C. on right annihilators.

Proof. Let $0 \neq I \triangle N$ and consider the collection of right annihilators $\{r(F)\}$ where $F$ runs over all finite subsets of $l$. From our assumption, there exists a minimal element $N=r\left(F_{0}\right) . M=(0)$, for if $M \neq 0$, then there is $0 \neq m \in M$ such that $F_{0} m=0 . N$ is strictly prime, hence there exists $0 \neq b \in N$ such that $m b m \neq 0$. Hence $b m \neq 0$. Let $S=r\left(F_{i} \cup\{b\}\right)$. Now $m \in M$ but $m \notin S$. Consequently $S \nsubseteq M$. The fact that $S$ is smaller than $M$ implies that $M=(0)$. Hence $N$ is strongly prime.

Theorem 2.5. Let $N$ be a zero symmetric near-ring. If $A$ is an ideal of $N$ and $P$ a strongly prime ideal of $N$, then $P \cap A$ is a strongly prime ideal of $A$.

Proof. Let $p \notin P \cap A$. Since $P$ is a strongly prime ideal in $N$, there exists a finite subset $F=\left\{x_{1}, \ldots, x_{n}\right\} \subseteq N$ such that if $p F c \subseteq P$, then $c \in P$. Let $d \in A$ such that $d \notin P$. It is now possible to find $x_{j} \in F$ such that $p x_{j} d \notin P$. For this $p x_{j} d \notin P$ we can find a finite set $F_{1} \subseteq N$ such that for all $t \in A, t \notin P$ we have $p x_{j} d F_{1} t \subseteq P$. Now $F_{2}=p x_{j} d F_{1}$ is a finite 
subset of $\langle p\rangle_{A}$, ideal generated by $P$ in $A$, such that for all $a \in A, F_{2} a \subseteq P \cap A$ implies $a \in P \cap A$. Hence $P \cap A$ is a strongly prime ideal in $A$.

Corollary 2.6. Let $M$ be the class of all zero symmetric strongly prime near-rings. $M$ is hereditary.

Proof. This follows from Theorem 2.5 if we take $P=(0)$.

An ideal $I$ of $N$ is called an essential ideal in $N$ if $I \cap K \neq \phi$ for any $0 \neq K \triangleleft N$. In this case we shall write $I \triangleleft \cdot N$.

Lemma 2.6. Let $M$ be any class of strongly prime near-rings. $M$ is closed under essential extensions.

Proof. Let $I \triangleleft \cdot N$ where $I \in M$. Furthermore, let $J$ be any nonzero ideal of $N$. Since $0 \neq I \cap J \triangleleft I$ and $I \in M$, there exists a finite subset $F \subseteq J \cap I$ such that $\{r \in I: F r=0\}=0$. We have $l(I) \triangleleft N$ where $l(I)=\{n \in N: n I=0\}$, i.e. left annihilator of $I$ in $N$. Furthermore, $[l(I) \cap I]^{2} \subseteq l(I) \cdot I=0$. Now, $l(I) \cap I \sqcup I$ and since $I$ is a prime near-ring, it follows that $l(I) \cap I=0$. From the fact that $I$ is an essential ideal in $N$, we have $l(I)=0$. Hence for all $0 \neq n \in N$ there exist $0 \neq p \in I$ such that $n p \neq 0$ and consequently, $F n p \neq 0$. From this we have $F n \neq 0$. Hence $N$ is strongly prime which proves the lemma.

Definition (see [6, p. 57]). Let $X$ be a homomorphically closed class of near-rings. A class of $\sigma$ of near-rings is called $X$-special if the following conditions are satisfied.

$S 1$ Each near-ring from $\sigma$ is prime.

$S 2 N \in \sigma \cap X$ and $A \triangleleft N$ implies $A \in \sigma$.

$S 3$ If $I \triangleleft A \triangleleft N \in X$ and $A / I \in \sigma$, then $I \triangleleft N$ and $N /(I: A)_{N} \in \sigma$ where $(I: A)_{N}=\{n \in N: n A \subseteq I\}$.

Let $D$ denote the class of all d.g. near-rings.

Theorem 2.8. The class $M$ of strongly prime near-rings is D-special.

Proof. From 2.3 and Corollary 2.6 it follows that conditions $S 1$ and $S 2$ are satisfied. We only have to show that $S 3$ is also satisfied. Let $I \triangleleft A \triangleleft N \in D$ and $A / I \in M$. Since $A / I$ is also a prime near-ring, it follows from [6, Theorem 5.3] that $I \triangle N$. We show that $N /(I: A)_{N} \in M$, i.e. that $(I: A)_{N}$ is a strongly prime ideal in $N$. Let $x \notin(I: A)$. There exists $a \in A$ such that $x a \notin I$. Since $I$ is a strongly prime ideal in $A$, there exists a finite subset $F$ of $\langle x a\rangle_{A} \subseteq\langle x\rangle_{N}$ such that for all $z \in A, F z \subseteq I$ implies $z \in I$. Let $t \in N$ be arbitrary such that $F t \subseteq(I: A)$. Since $F t A \subseteq I$, we have $t A \subseteq I$. Hence $t \in(I: A)$. Therefore $(I: A)_{N}$ is a strongly prime ideal in $N$ and consequently $N /(I: A)_{N} \in M$.

\section{The strongly radical prime}

Definition. Let $N$ be any near-ring. As in the case of rings [2], we define the strongly prime radical $s(N)$ of $N$ as the intersection of all the strongly prime ideals in $N$. 
If $M$ is the class of all strongly prime near-rings, let $U M$ be the upper radical class determined by $M$. The following result is easy to prove.

Proposition 3.1. If $M$ denotes the class of all strongly prime near-rings, then $U M=\{N: N$ is a near-ring such that $N=s(N)\}$.

We also need the following result which follows from [1] and [10].

Proposition 3.2. Let $M$ be a regular and essential closed class of near-rings satisfying the following condition:

$$
I \triangleleft K \triangleleft N \text { and } I / K \in M \text { imply } K \triangleleft N .
$$

Then $U M(N)=\cap\left\{I_{a} \triangleleft N: N / I_{a} \in M\right\}$.

Proof. This follows from [10, Proposition 11] and [1, Theorem 1].

Theorem 3.3. If $M$ is the class of all strongly prime near-rings, then for every $N \in D$ and $I \triangleleft N$ we have $U M(I)=I \cap U M(N)$ and $s(N)=U M(N)$.

Proof. From Theorem 2.8 and Lemma 2.7 it follows that the conditions of [1, Theorem 1] are satisfied. From [1, Theorem 1] we have $U M$ hereditary and from [1, Proposition 3] it follows that $S U M$ is hereditary. Hence for every $N \in D$ and every ideal $I$ of $N$ we have $U M(I)=U M(N) \cap I$.

Since $M \in D$ is a regular and essentially closed class of near-rings satisfying condition $(F)$ we have $U M(N)=s(N)$ for each $N \in D$.

Remark. In general, $s(N) \cap I \neq s(I)$ where $I \triangleleft N$. Take $N$ and $I$ as in Example 1. $I$ is a strongly prime ideal in $N$ : For every $x \notin I$ take $F=\{4\} \subseteq\langle x\rangle=N$. Now we have $F y \nsubseteq I$ for each $y \notin I$. Since (0) is not a strongly prime ideal we have $s(N)=I=\{0,1,2,3\}$. We show $K=\{0,2\}$ is a strongly prime ideal in $I$. For each $x \notin K$, take $F=\{1\} \subseteq\langle x\rangle=I$. Now $F y \nsubseteq K$ for each $y \notin K$. Hence $K$ is a strongly prime ideal in $I$. Since $I$ is not a prime near-ring it is also not a strongly prime near-ring. Hence $s(I)=K$ and $K=s(I) \nsubseteq s(N) \cap I=I$.

Regarding the position of strongly prime radical among the well-known radicals, we have: If $P(N)$ denotes the prime radical of the near-ring $N$, then we have from Lemma 2.3 that $P(N) \subseteq s(N)$. This inclusion can be strict, for the near-ring in Example 1 is prime. Hence $P(N)=0 \nsubseteq I=s(N)$. In [4] the completely prime radical of a near-ring $N, C(N)$, was defined as the intersection of all the completely prime ideals, i.e. all ideals $I \triangleleft N$ such that $a, b, \notin I$ implies $a b \notin I$. Every completely prime ideal is strongly prime for if $I$ is completely prime then for every $x \notin I$ take $F=\{x\}$. Clearly $F y \nsubseteq I$ for every $y \notin I$. Hence $s(N) \subseteq C(N)$.

We have the following example to show that this inclusion can be strict.

Example 2. Let $N$ be the ring of $2 \times 2$ matrices over the two element field $Z_{2}=[0,1]$. Every nonzero ideal of $N$ contains the one element subset $F=\left[\begin{array}{ll}1 & 0 \\ 0 & 1\end{array}\right]$ such that $F z=0$ 
implies $z=0, z \in N$. Hence $N$ is strongly prime and therefore $s(N)=(0) . N$ is not completely prime for $0 \neq\left[\begin{array}{ll}0 & 1 \\ 0 & 0\end{array}\right]$ and $0 \neq\left[\begin{array}{ll}1 & 0 \\ 0 & 0\end{array}\right]$ but $\left[\begin{array}{ll}0 & 1 \\ 0 & 0\end{array}\right]\left[\begin{array}{ll}1 & 0 \\ 0 & 0\end{array}\right]=\left[\begin{array}{ll}0 & 0 \\ 0 & 0\end{array}\right]$. Hence $C(N)=N$ and consequently $s(N) \nsubseteq C(N)$.

In [3] it was proved that the Levitzki radical $L(N)$ of a near-ring $N$ is the intersection of all the $l$-prime ideals of $N . I \triangleleft N$ is a $l$-prime ideal if for every $a \notin I$ there exists a finite number of elements $a_{1}, a_{1}, \ldots, a_{n(a)} \in\langle a\rangle$ such that the following condition is satisfied. If $a, b \notin I$ then for every $n>1$ there exists a product of $N \geqq n$ factors, consisting of $a_{i}$ 's and $b_{j}$ 's which is not in $I$.

Lemma 3.4. If $N$ is a near-ring, then $L(N) \subseteq s(N)$.

Proof. We show that any strongly prime ideal is a $l$-prime ideal. Suppose $I \Delta N$ is a strongly prime ideal in $N$. For every $a, b, \notin I$ there exists finite sets $F_{1} \subseteq\langle a\rangle$ and $F_{2} \subseteq\langle b\rangle$ such that for any $x \notin I$ we have $F_{1} x \subseteq I$ and $F_{2} x \subseteq I$. It is now easy to show that for every $n>1$ there exists a product of $N \geqq n$ factors consisting of elements from $F_{1}$ and $F_{2}$ which is not in $I$. Hence $I$ is an l-prime ideal and therefore $L(N) \subseteq s(N)$.

For a characterization of $s(N)$ by using certain systems (as is the case for the prime radical and $m$-systems) we use the approach of [11] for rings.

Definition. An sp-system in $N$ is a pair $(G, P)$ where $P$ is an ideal in $N$ and $G$ is a subset of $N$ such that $G \cap P$ contains no nonzero elements of $N$ and for any $g \in G$, there is a finite subset $F \subseteq\langle g\rangle$ such that $F z \cap G \neq \phi$ for all $z \notin P$.

Now $I \triangleleft N$ is a strongly prime ideal if and only if $(N \backslash I, I)$ is an sp-system.

Proposition. For any near-ring $N$, we have $s(N)=\{x \in N$ : if $x \in G$ where $(G, I)$ is an sp-system for some ideal $I$ in $N$, then $0 \in G$ \}.

Proof. The same as for rings (cf. [11, Proposition 2.3]).

Acknowledgement. The author wishes to record here his thanks to the referee for helpful and constructive comments.

\section{REFERENCES}

1. T. Anderson, K. KaArli and R. Wiegandt, Radicals and subdirect decomposition, preprint.

2. N. J. Groenewald and G. A. P. Heyman, Certain classes of ideals in group-rings. Communications in Algebra 9 (1981), 137-148.

3. N. J. Groenewald and P. C. Porgieter, A note on the Levitzki radical of a near-ring, J. Austral. Math. Soc. (Series A) 36 (1984), 416-420.

4. N. J. Groenewald, The completely prime radical in near-rings, Acta Math. Hungar. (to appear).

5. D. Handelman and J. Lawrence, Strongly prime rings, Trans. Amer. Math. Soc. 211 (1975), 209-223.

6. K. KaARLI, Special radicals of near-rings (in Russian), Tartu Riikl. Ul. Toimetised Vih. 610 (1982), 53-68. 
7. A. Oswald, Some topics in the structure theory of near-rings (Doctoral Dissertation, University of York, 1973).

8. M. M. Parmenter, P. N. Stewart and R. Wiegand, !On the Groenewald-Heyman strongly prime radical, Quaestiones Math. 7 (1984), 22-240.

9. G. PlLz, Near-Rings (North-Holland Mathematical Studies, 1977).

10. L. C. A. van Leeuwen and R. Wiegandt, Semisimple and torsion-free classes, Acta Math. Academica Scientiarum Hungaricae Tomus 38 (1981), 73-81.

11. S. Veldsman, The elements in the strongly prime radical of a ring, preprint.

Department of Mathematics

University of Port Elizabeth

PO Box 1600

6000 Port Elizabeth

SOUTh Africa 